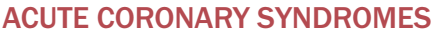

\section{Postconditioning reduces myocardial edema}

Ischemic postconditioning - the application of repeated, brief periods of ischemia at the time of reperfusing a coronary artery after an ST-segment elevation myocardial infarction (STEMI) - is known to reduce infarct size, and has now been shown, for the first time in humans, also to reduce myocardial edema. "Edema, which can be measured by cardiac MRI, might become a marker of ischemia-reperfusion injury," says Professor Michel Ovize from Lyon, France, and a lead investigator in this research study.

The beneficial restoration of blood flow during reperfusion therapy can, paradoxically, also be associated with injurious changes in oxidative stress, intracellular calcium levels, $\mathrm{pH}$, and inflammation. Concomitant myocardial edema, which begins during ischemia, can acutely worsen during reperfusion and cause cell damage.

Between May 2008 and October 2010, the investigators recruited 50 patients with STEMI who received standard stenting by primary percutaneous coronary

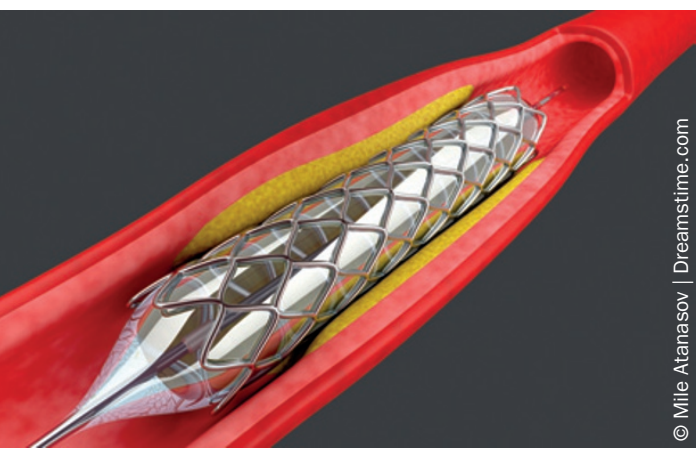

intervention. Half the patients were then randomly allocated to have no additional intervention, while the other half received postconditioning. Within $1 \mathrm{~min}$ of stent deployment and reflow in the postconditioned patients, the angioplasty balloon was reinflated for the first of four 1 min periods, each separated by $1 \mathrm{~min}$ of reflow. At baseline, no significant differences were observed between the patients, and the mean size of the area at risk, measured by coronary angiography, was comparable between the two groups.

Postconditioning was associated with a $38 \%$ reduction in the mean infarct size, measured by late-gadolinium enhancement MRI, compared with controls $\left(13 \pm 7 \mathrm{~g} / \mathrm{m}^{2}\right.$ versus $\left.21 \pm 14 \mathrm{~g} / \mathrm{m}^{2} ; P=0.01\right)$. Similarly, the extent of myocardial edema, measured by T2-weighted MRI, was 32\% lower in postconditioned patients than controls $\left(23 \pm 16 \mathrm{~g} / \mathrm{m}^{2}\right.$ versus $\left.34 \pm 18 \mathrm{~g} / \mathrm{m}^{2} ; P=0.03\right)$.

We now intend "to look for the optimal way to assess the area at risk in STEMI patients (not involving edema-based techniques ... because, as demonstrated indirectly in our paper, any treatment that reduces infarct size also limits edema). We [also] plan to address the issue of the role of edema as a cause of infarct expansion during the reperfusion phase," says Professor Ovize.

Gregory B. Lim

Original article Thuny, F. et al. Post-conditioning reduces infarct size and edema in patients with ST-segment elevation myocardial infarction. J. Am. Coll. Cardiol. 59, 2175-2181 (2012) 